\title{
Sequential Coronal Mass Ejections from AR8038 in May 1997
}

\author{
Y. Li • B.J. Lynch • B.T. Welsch • G.A. Stenborg • \\ J.G. Luhmann • G.H. Fisher · Y. Liu • R.W. Nightingale
}

Received: 30 March 2009 / Accepted: 24 March 2010 / Published online: 20 April 2010

(C) The Author(s) 2010. This article is published with open access at Springerlink.com

\begin{abstract}
Three homologous coronal mass ejections (CMEs) occurred on 5, 12 and 16 May 1997 from the single magnetic polarity inversion line (PIL) of AR8038. The three events together provide STEREO-like quadrature views of the 12 May 1997 CME and EIT double dimming. The recurrent CMEs with the nearly identical post-CME potential state and the 'sigmoid to arcade to sigmoid' transformations indicate a repeatable store - release - restore process of the free energy. How was the free magnetic energy re-introduced to the potential state corona after each release in this decaying active region? Making use of the known time
\end{abstract}

Electronic supplementary material The online version of this article

(doi:10.1007/s11207-010-9547-y) contains supplementary material, which is available to authorized users.

Y. Li (₫) · B.J. Lynch · B.T. Welsch · J.G. Luhmann · G.H. Fisher

Space Sciences Laboratory, University of California, Berkeley, CA, USA

e-mail: yanli@ssl.berkeley.edu

B.J. Lynch

e-mail: blynch@ssl.berkeley.edu

B.T. Welsch

e-mail: welsch@ssl.berkeley.edu

J.G. Luhmann

e-mail: jgluhman@ssl.berkeley.edu

G.H. Fisher

e-mail: fisher@ssl.berkeley.edu

G.A. Stenborg

Interferometrics, Inc., 13454 Sunrise Valley Dr., Herndon, VA 20171, USA

e-mail: guillermo.stenborg@nrl.navy.mil

Y. Liu

Hansen Experimental Physics Laboratory, Stanford University, Stanford, CA, USA

e-mail: yliu@solar.Stanford.edu

R.W. Nightingale

Lockheed Martin Advanced Technology Center, Department L9-41, Building 252, Palo Alto, CA, USA

e-mail: nightingale@Imsal.com 
interval bounded by the adjacent homologous CMEs, we made the following measures. The unsigned magnetic flux of AR8038, $\Phi_{\mathrm{AR}}$, decreased by approximately $18 \%$ during $66 \mathrm{~h}$, while the unsigned flux, $\Phi_{\mathrm{PIL}}$, in a Gaussian-weighted PIL-region containing the flare site increased by about $50 \%$ during 36 hrs prior to the C1.3 flare on 12 May 1997. The significant increase of $\Phi_{\mathrm{PIL}}$ demonstrates the magnetic gradient increase and the build-up of free energy in the PIL-region during the time leading to the eruption. Fourier local correlation tracking (FLCT) flow speed in AR8038 ranges from 0 to $292.8 \mathrm{~m} \mathrm{~s}^{-1}$ with a mean value of $63.2 \mathrm{~m} \mathrm{~s}^{-1}$. The flow field contains a persistent converging flow towards the flaring PIL and an effective shear flow distributed in the AR. Minor angular motions were found. An integrated proxy Poynting flux $S_{\mathrm{h}}$ estimates the energy input to the corona to be on the order of $1.15 \times 10^{32}$ erg during the $66 \mathrm{hrs}$ before the $\mathrm{C} 1.3$ flare. It suggests that sufficient energy for a flare/CME can be introduced to the corona on the order of several days by the flows deduced from photospheric magnetic field motions in this small decaying active region.

Keywords Coronal mass ejection · EUV dimming · Flare · Magnetic field · Sigmoid

\section{Introduction}

Solar eruptions are processes that rapidly release free magnetic energy or non-potential magnetic energy in the corona. Eruptions may originate from emerging or decaying active regions (ARs) and well-decayed magnetic regions with quiescent filaments. In the case of emerging ARs, the free magnetic energy carried by electric current emerges from the solar interior where solar dynamo is operating (Leka et al., 1996; Fisher et al., 2000; Schrijver, 2007; Welsch and $\mathrm{Li}, 2008$ ). In decaying ARs or decayed magnetic regions, it becomes arguable whether the free energy was generated below the photosphere or above it by flows, magnetic flux cancellation and other processes (Forbes et al., 2006; Welsch, 2006). In addition to the energy source, it has been suggested that the coronal magnetic topology may play a role in CME initiation (Antiochos, DeVore, and Klimchuk, 1999; Lynch et al., 2008). Eruptions appear to be random in nature apart from the general solar activity cycle dependence. The energy build-up process and the initiation of the eruptions are currently outstanding questions, despite significant progress in the past decade combining observational and numerical modeling/simulation efforts.

Numerous studies have been devoted to the 12 May 1997 flare and halo coronal mass ejection (CME) from AR8038 (Plunkett et al., 1998; Thompson et al., 1998; Hudson et al., 1998; Webb et al., 2000; Mathew and Ambastha, 2000; Li et al., 2004; Liu, 2004; Crooker and Webb, 2006; Titov et al., 2008). This event was one of a few halo CMEs early in the SOHO mission, with a fairly complete set of data including solar, heliospheric and geo-space observations. The Sun was magnetically simple at the time shortly after solar minimum and the AR itself was a small classic bipolar region with a positive leading polarity bearing a sunspot and a defuse negative polarity (a new-cycle sunspot). The symmetric halo CME was associated with the best-known symmetric EIT double dimming, an EIT wave with a circular wave front and a C1.3 X-ray flare. The counter-part ICME containing a magnetic cloud was observed in-situ on 15 May at the L1 point by the Wind satellite (Webb et al., 2000; Arge et al., 2004). The 12 May halo CME has been a focused event for many coordinated research efforts. It was a target case for the NSF SHINE group with observational and MHD modeling studies. It is also an NSF CISM event for coordinated solar, heliospheric and geospace modeling effort. Yet, the initiation process of this seemingly simple solar eruption is still not fully understood. 
Previous observational studies using magnetic field data of this event had focused on the onset of the flare, associations between magnetic signatures and the eruption, and/or changes before and after the eruption on 12 May. Mathew and Ambastha (2000) studied magnetic signatures related to the onset of the C1.3 flare on 12 May by following several small individual magnetic features/fragments near the polarity inversion line (PIL) of AR8038 during 10 to 13 May. They reported magnetic flux cancellation and emergence associated with moving magnetic features at speeds ranging $300-800 \mathrm{~m} \mathrm{~s}^{-1}$. They noted an intermittent local gradient increase at the site of initial flare brightening (feature marked as $6 \mathrm{p}$ in their Figure 5). Although they have a magnetogram sequence of four days, they calculated the magnetic flux of a small rectangular area at the flare site enclosing feature $6 \mathrm{p}$ to show a flux reduction at three time points around the flare. Liu (2004) found both small and large scale magnetic field changes before and after the 12 May flare/CME. He also reported flux cancellation and emergence of magnetic features near the PIL. Li et al. (2004) reported converging flow towards the PIL and the temporal evolution of the flow along a single perpendicular cut through the center of the sunspot using their preliminary flow field obtained by applying Fourier local correlation tracking (FLCT) (Welsch et al., 2004, 2007; Fisher and Welsch, 2008 ) to the line of sight (LOS) magnetic field without projection corrections. LCT techniques measure magnetic field-related flow field from a different perspective than magnetic feature tracking (further description in Section 3). The results of LCT have the potential to either inspire or serve as the input to numerical modeling for data-driven CME predictions. The FLCT method and pre-processing of magnetograms have been improved in the last few years in our group (Welsch et al., 2007, 2009; Fisher and Welsch, 2008). Further analysis and updated results are discussed later in this paper.

Titov et al. (2008) constructed a simple model of current-free coronal magnetic field by superimposing an observation-based large scale background field and a localized bipolar AR field as an analog to the magnetic field on 12 May 1997. They then evolved the modeled magnetic field to study pre-eruption evolution of the structure by applying vortex motions to the two flux concentration of the AR to shear the initially potential field to a force-free field, upon which flux cancellation is enabled to further evolve the field as inspired by observations. They showed that a fluxrope is formed in the flux cancellation phase but no attempt is made to erupt the fluxrope in this simple model. A global MHD simulation of the 12 May CME based on a MDI synoptic map has successfully initiated the eruption; some of their results were presented at AGU meetings (Linker et al., 2006, 2008) and a paper including full details of their work is currently in preparation (J. Linker, personal communication).

We found a sequence of three CMEs that occurred several days apart from AR8038, including the 12 May halo CME. Thus, this well-studied CME may in fact be one of three recurrent events, which would put more constraints on the energy build-up and initiation process, in contrast to previous studies of the 12 May CME as a single eruption. For this purpose, we need to first establish that the three CMEs are homologous (similar) by analyzing coronal imaging data in white light, EUV and soft X-ray wavelengths. Given that the CMEs are homologous, the two CMEs near the limbs could also effectively provide views from an orthogonal perspective of a CME structure, adding to our existing knowledge of the 12 May CME. Particularly essential to this study, these recurrent CMEs from the same magnetic source may experience the same magnetic energy build-up and release process. The eruption on 5 May serves to pre-condition the eruption source region, in that the free energy needed for a similar eruption is released at a known point of time and a new cycle of energy build-up begins then from a potential or near potential state. Our study intends to contribute to the further understanding of the connection between magnetic field evolution and solar eruptions, and to the effort of improving our capability to predict CMEs. 

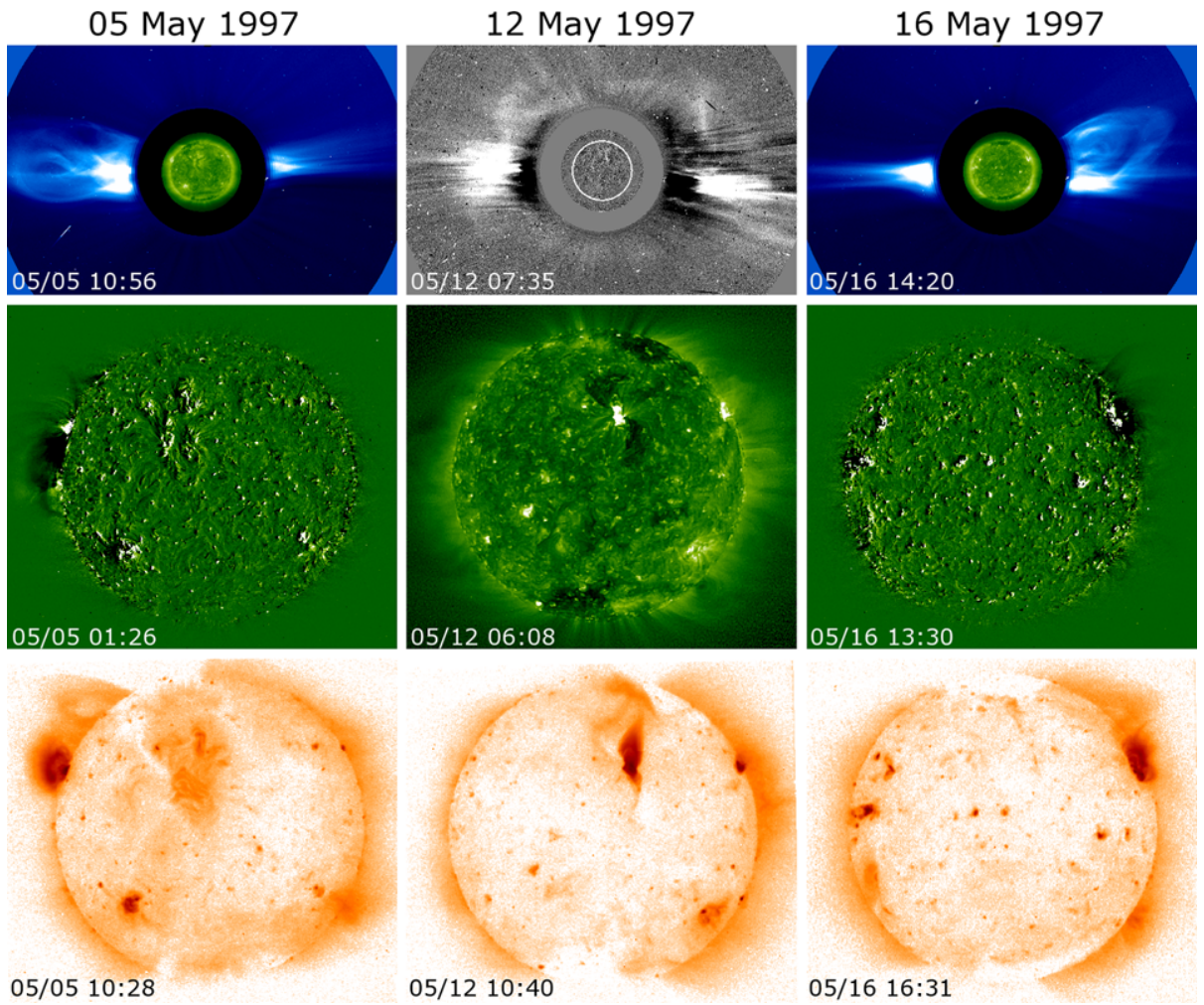

Figure 1 Three major eruptions from the same magnetic polarity inversion line (PIL) in AR8038. Top row: LASCO coronagraph images of CMEs on 5, 12 and 16 May, from left to right. A difference image is used for the halo CME on 12 May. Middle row: EIT difference images and a wavelet enhanced image (12 May) for the three eruptions. Double EUV dimmings are observed for the CMEs (see attached movies: eit19970505difmv.gif, eit19970512mv.gif, and eit19970516difmv.gif). Bottom row: Yohkoh soft X-ray images show similar post-eruption arcades and candle-flame cusps (see attached movies: sxt19970505mv.gif, sxt19970511mv.gif and sxt19970516mv.gif).

\section{Three Sequential CMEs: Homologous Events?}

\subsection{Overview}

Three CMEs occurred on 5, 12 and 16 May 1997 from the same magnetic polarity inversion line (PIL) in AR8038, when the source region was at the east limb, near the central meridian, and near the west limb, respectively. Figure 1 gives an overview of the three eruptions, with LASCO coronagraph images and a difference image (halo CME on 12 May) in the top row, EIT difference images and a wavelet enhanced image (on 12 May) in the middle row and Yohkoh SXT (soft X-ray Telescope) images in the bottom row. Table 1 summarizes the observational signatures associated with the CME sequence and gives the location of the AR at the time of the eruptions. Some of these quantities are adapted from the LASCO CME catalog, GOES flare catalog, active region maps archive by the Institute of Astronomy of the University of Hawaii and the published literature. The CME time refers to its first appearance in the LASCO C2 field of view. All three CME speeds given in Table 1 are measured in the plane-of-the-sky. The frontal speed of the halo CME on 12 May was estimated to be about 
Table 1 Three major eruptions from AR8038 during 5 to 17 May 1997.

\begin{tabular}{|c|c|c|c|}
\hline Date & 5 May & 12 May & 16 May \\
\hline CME time (C2) & $6: 30 \mathrm{UT}$ & $5: 30 \mathrm{UT}$ & $\approx 13$ UT (data gap) \\
\hline Speed (pos) & $395 \mathrm{~km} \mathrm{~s}^{-1}$ & $464 \mathrm{~km} \mathrm{~s}^{-1}$ & $554 \mathrm{~km} \mathrm{~s}^{-1}$ \\
\hline Geometry & PA:87 AW:55 & Halo & PA:276 AW:49 \\
\hline EUV dimmings & Double dimming & Double dimming & Possible double dimming \\
\hline Activation & 00:43 UT & 04:50 UT & Timeline unclear \\
\hline Formed & 01:26 UT & 05:07 UT & \\
\hline Duration & $6 \mathrm{~h} 34 \mathrm{~min}$ & $13 \mathrm{~h} 23 \mathrm{~min}$ & \\
\hline EUV wave & $\begin{array}{l}\text { no clear } \\
\text { wave front }\end{array}$ & $\begin{array}{l}245 \pm 40 \mathrm{~km} \mathrm{~s}^{-1} \\
\text { circular } \\
\text { wave front }\end{array}$ & $\begin{array}{l}\text { no clear } \\
\text { wave front }\end{array}$ \\
\hline GOES X-ray flare & B 4.3 & $\mathrm{C} 1.3$ & B2.1 \\
\hline Start & 00:35 UT & 04:42 UT & 12:56 UT \\
\hline Max & 01:01 UT & 04:55 UT & 13:01 UT \\
\hline End & 01:31 UT & 05:26 UT & 13:07 UT \\
\hline AR8038 location & N20E80 & N21W09 & N20W65 \\
\hline
\end{tabular}

$600 \mathrm{~km} \mathrm{~s}^{-1}$ after correcting for the projection effects (Plunkett et al., 1998; Zhao, Plunkett, and Liu, 2002). All three CMEs had moderate speeds. Both the east limb 5 May CME and the west limb 16 May CME have the classic three-part CME white-light structure composed of a bright loop front, a darker cavity and a bright core. The 12 May CME is directed approximately along the Sun-Earth line and appears as a symmetric halo surrounding the Sun. We will now examine the three-dimensional EIT dimming structure, the SXT sigmoid evolution and the global coronal field structure to show that this sequence of CMEs can be considered homologous events.

\subsection{EUV Dimming}

The EUV double dimmings along with the 12 May halo CME are the best-known examples of the phenomena. Symmetric double dimmings are rarely observed but attracted a lot of attention and research effort because they well represent the common interpretation, i.e., that the two dimming regions are the footprints of an expanding fluxrope still connected to the Sun and experiencing significant density depletion (Thompson et al., 1998; Webb et al., 2000; Yurchyshyn et al., 2006; Liu et al., 2007; Attrill et al., 2008). However, since we have only one view of a 3D density depletion structure, both the EUV dimming structure and the connection between EUV dimming and CME fluxrope are not clearly understood. Attempts to compare the magnetic flux in the EUV dimming regions with the corresponding in-situ ICME magnetic fluxropes have shown some, albeit moderate, success (Webb et al., 2000; Qiu et al., 2007; Attrill et al., 2008).

Using a sequence of wavelet-enhanced EIT images and EIT difference images, we found that the CMEs on 5 and 16 May have also EIT double dimmings similar to the 12 May case (see attached animations eit19970505difmv.gif, eit19970512mv.gif and eit19970516difmv.gif). The wavelet image processing is used to reveal coronal fine structure in much greater clarity. 

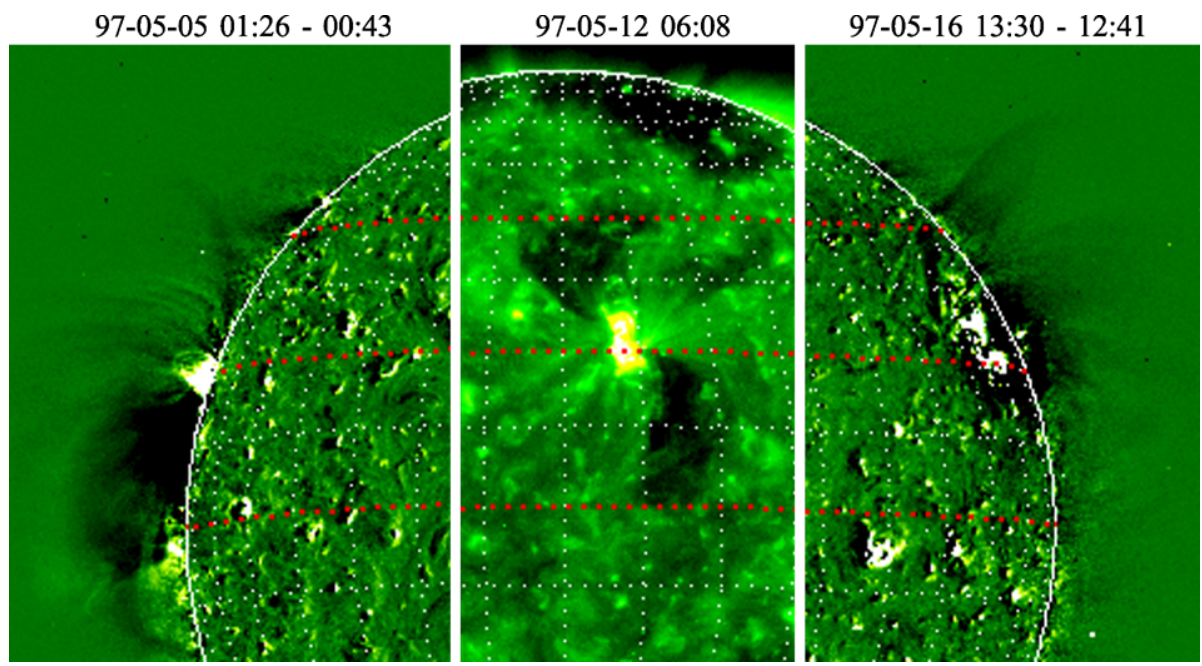

Figure 2 Zoom-in view of the dimming regions of the three eruptions are shown with the latitude grid aligned. The latitude grids are spaced by $10^{\circ}$, and latitudes $0^{\circ}, 20^{\circ}$ and $40^{\circ}$ are highlighted in red color.

Its application to SOHO EIT data has been described by Stenborg, Vourlidas, and Howard (2008).

If the EUV dimmings associated with these CMEs are sufficiently similar, we can consider them as analogous of STEREO-like views of the 12 May double dimming in threedimensions. Figure 2 includes the EUV dimming regions of the three CMEs. The latitude and longitude grids on the solar surface are shown as dotted lines spaced at $10^{\circ}$ intervals. The $0^{\circ}, 20^{\circ}$, and $40^{\circ}$ latitudes are highlighted in red to show that the latitudinal extent of the 12 May double dimmings lines up reasonably well with the extent of both the east and west limb dimming structures. The dimmings on the limbs are much less obvious due to low contrast to the sky background intensity and are better shown with difference images.

The east limb dimmings show a similar morphology to the 12 May twin dimmings but is less symmetric in intensity with the southern part more pronounced. Note that if the dimming regions are inclined to the local meridian similar to the 12 May event, more emission might be in the forefront of the northern part. The east limb dimming regions show connection with the solar disk at the small tip areas close to the flaring AR, and most of the volume of the dimming structure is elevated in the corona above the disk. The dimming 'lobes' have clear boundaries in the corona reaching about $1.2 R_{\odot}$. There is no apparent connection between the two dimming 'lobes' in the EIT field of view. However, the limited coverage of the solar atmosphere above the limb and some brighter emission in the forefront could prevent one from observing the complete structure of the dimming. The west limb 16 May double dimming appears less structured than its east limb counterpart, but shares the basic properties.

These observations show similar dimming structures for the three CMEs. They are, however, insufficient to either support or reject the most commonly accepted interpretation that double dimmings are the footprints of a single fluxrope. The STEREO-like views of the dimmings do support the interpretation that the majority of the dimming areas on the solar disk on 12 May are the 'projections' of elevated structures in the corona above, and the temporary open magnetic flux is from some small fraction of the dimming areas near the AR or the darker cores of the dimmings (Webb et al., 2000). 


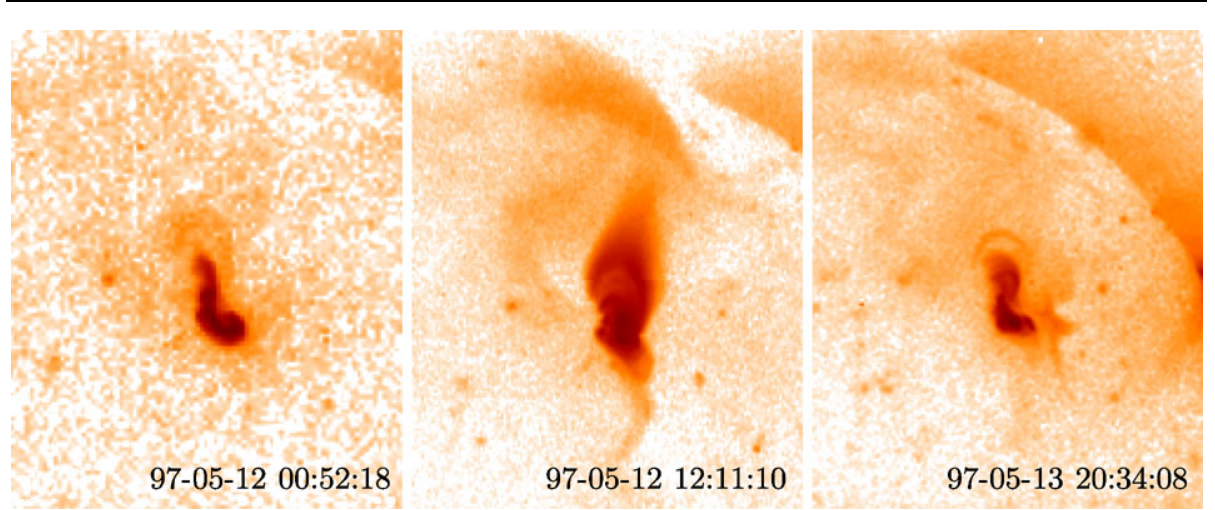

Figure 3 The SXR 'sigmoid to arcade to sigmoid' transformations before and after the eruption on 12 May. See attached SXR movie (sxt19970511mv.gif) for the process.

A spectacular EUV wave was also generated by the 12 May eruption. This global-scale disturbance had a circular wave front with an average propagation speed of $245 \pm 40 \mathrm{~km} \mathrm{~s}^{-1}$ (Thompson et al., 1998). We found no identifiable wave fronts along with the 5 and 16 May limb CMEs (see attached animations eit19970505difmv.gif, eit19970512wave.gif, and eit19970516difmv.gif).

\subsection{SXR Sigmoid Evolution}

Coronal soft X-ray (SXR) sigmoidal structure represents sheared magnetic field with free energy and is one of the most recognized pre-eruptive features (Canfield, Hudson, and McKenzie, 1999; Canfield, Hudson, and Pevtsov, 2000; Moore et al., 2001; Green and Kliem, 2009) in addition to solar prominences/filaments. During an eruption, a sigmoid is transformed in the pattern termed the 'sigmoid to arcade' signature (Sterling et al., 2000; Canfield, Hudson, and Pevtsov, 2000; Moore et al., 2001), indicating the coronal structure changes from a sheared to a less-sheared (or more potential) magnetic configuration (Sterling et al., 2000; Liu, 2004).

For these three recurrent CMEs, the pre-eruption sigmoid and its evolution are best observed near the central meridian around the 12 May event, as shown in Figure 3. The images show the pre-flare sigmoid (00:52:18 UT), the post-flare arcade and cusp (12:11:10 UT), and the 'rebuilt' sigmoid (20:34:08 UT 13 May) from left to right, respectively. After the transformation of sigmoid to potential-like arcade by the eruption, another sigmoid at the same flaring PIL soon begin to appear and reformed in about 40 hours to a similar state with the previous one. The two limb events show similar post-eruption arcade and cusp (see attached animations: sxt19970505mv.gif, sxt19970511mv.gif, and sxt19970516mv.gif).

The 'sigmoid to arcade' reconfiguration has been well discussed and understood as described above (Sterling et al., 2000; Canfield, Hudson, and Pevtsov, 2000; Moore et al., 2001; Liu, 2004). The 'arcade to sigmoid' transformation is less addressed. The 'sigmoid to arcade to sigmoid' transformations together suggest a magnetic free energy release and restore cycle. The earlier eruption released the stored free energy and set the source region to a potential state at a known point of time and the energy restore process for the next eruption begins thereafter. 

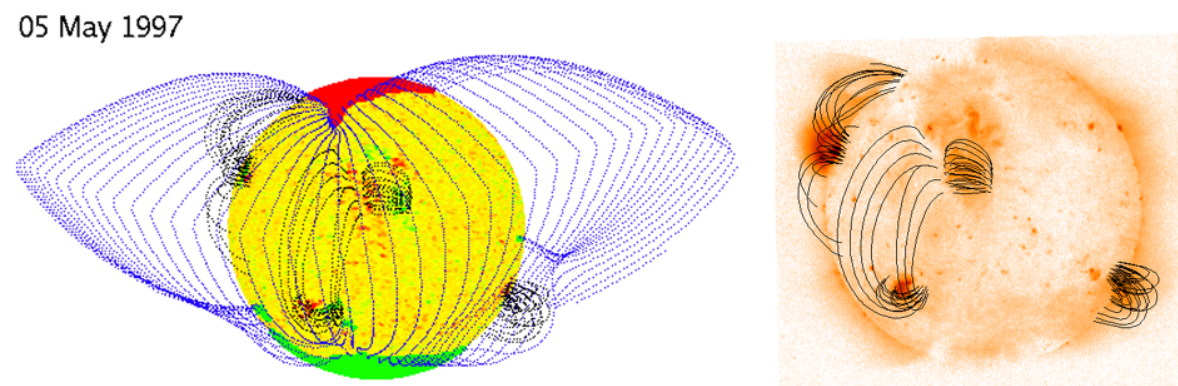

1997/05/05 10:28:38

12 May 1997
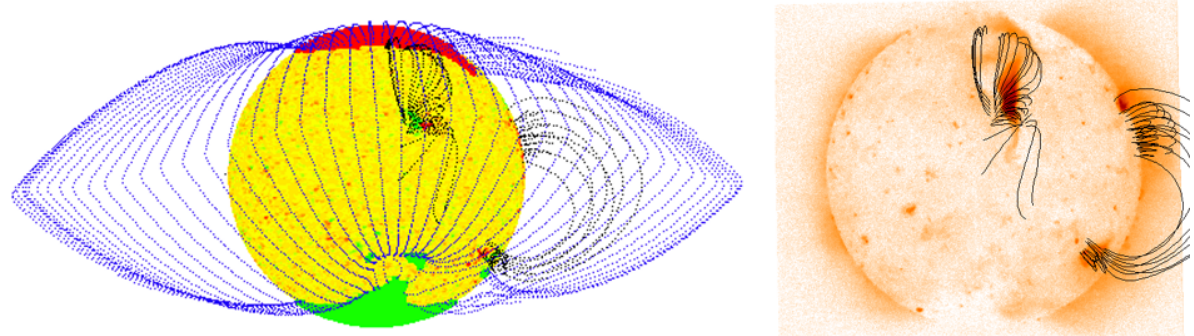

1997/05/12 08:56:52

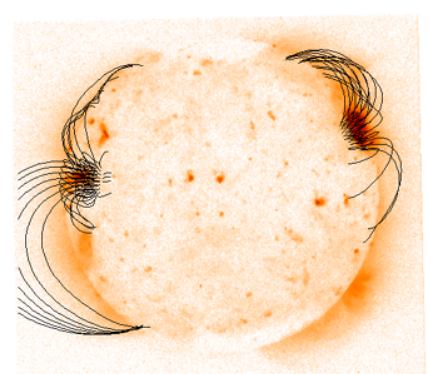

1997/05/16 14:34:56

16 May 1997

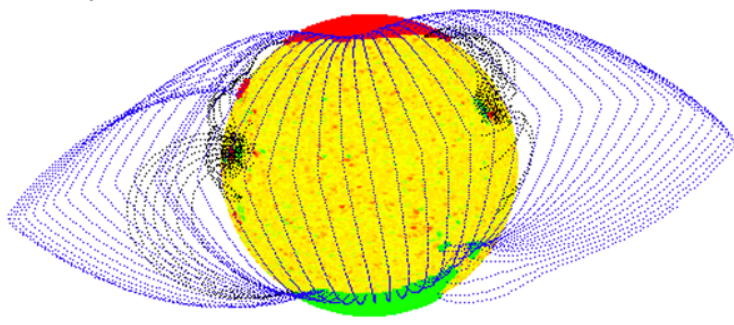

Figure 4 Left: PFSS streamer field and AR location for each of the three events. Right: AR field lines overlaid on soft X-ray post-eruption arcades.

\subsection{Global Coronal Magnetic Field Structure}

The coronal magnetic field is estimated through potential field source surface (PFSS) modeling (Li and Luhmann, 2006, and references therein) based on the Mt. Wilson Observatory (MWO) magnetogram synoptic map of Carrington rotation 1922 (25 April to 21 May 1997). AR8038 was the only strong magnetic region on a quiet solar disk during the early ascending phase of solar cycle 23, within a year of the sunspot minimum. The left column of Figure 4 shows the PFSS model results for the global coronal magnetic field from Earth view of the Sun on 5, 12, and 16 May in the top, middle, and bottom panels, respectively. The magnetic synoptic map is transformed onto the solar surface with positive (negative) polarity colored red (green). The open field regions of the polar coronal holes are colored solid red or green indicating their polarity on the surface. The boundary of the coronal helmet streamer belt is shown as a series of blue field lines traced from their apex just below $2.5 R_{\odot}$ (representing the outermost closed field lines). The PFSS coronal helmet streamer geometry compares favorably with the coronagraph observations. The coronal field shows a nearly dipolar mag- 
netic field geometry with a flat equatorial coronal helmet streamer belt, and the open fields from only the polar coronal holes.

The black field lines beneath the streamer belt are traced from the strong field regions in the closed field corona. In the right column of Figure 4 these same strong-field field lines are plotted over the Yohkoh soft X-ray (SXR) post-CME images in reverse color. Remarkably similar large scale bright SXR loops connecting the AR to the edge of the northern coronal hole are seen after each of the three CMEs. The PFSS field lines from AR8038 show good agreement with the location and geometry of these large scale post-CME SXR arcades. Previously, Liu (2004) showed a good agreement between potential field lines and postCME SXR arcades on the active region scale (see Figure 8 of Liu, 2004 for further details). Our findings in this section and the last section, together with previous work, indicate that the corona relaxed to a potential field state with nearly identical configurations and connectivity after the energy release by each eruption.

Given the similarities of the three eruptions and the reforming of a similar sigmoid after the 12 May post-eruption arcade, we conjecture that the three CMEs can be considered homologous events. Within this context, we now analyze the source region magnetic field evolution for the likely process that restores the free energy.

\section{Photospheric Magnetic Field Evolution in AR8038}

\subsection{Magnetic Flux Evolution}

MDI full disk magnetograms of LOS magnetic field with 2" pixel size and 96 min cadence (Scherrer et al., 1995) are analyzed for the magnetic flux evolution in AR8038. Figure 5 (left) gives a sample full disk magnetogram on 12 May 1997, where white (black) represents positive (negative) field. AR8038 was the only strong magnetic region and located at about $20^{\circ} \mathrm{N}$ and crossed the central meridian at about 12:30 UT on 11 May (see Table 1). A zoom-in magnetogram showing detailed flux distribution of AR8038 is given in the center panel, where black (white) cross symbol marks the positive (negative) weighted flux center (Welsch and Longcope, 2003). Figure 5 (right) shows a smoothed magnetogram of the region overlaid with selected contours to show the overall configuration of AR8038, where the solid white lines represent the zero-level contours. The long solid white contour line through the region is the polarity inversion line (PIL) of AR8038. The magnetic PIL is also commonly referred to as the magnetic neutral line (NL). We use the LOS magnetic field data in our analysis when AR8038 is within $45^{\circ}$ from the central meridian (LOS magnetic field data become less accurate closer to the solar limb). Thus, the interval under study is from 12 UT, 9 May to 12 UT, 13 May. The AR was mostly inactive during the time span between the two CMEs on 5 and 12 May. We assume the process to build the magnetic free energy driving the 12 May eruption begins after the one on 5 May. Although there are no adequate data immediately after the eruption on 5 May, the magnetic field evolution can be analyzed at least during about half of the time between the post-eruption potential state on 5 May and the next eruption on 12 May for some crude quantitative estimates.

We have recently developed routines using Mercator de-projection scheme for preprocessing of the magnetograms to compensate for foreshortening (Welsch et al., 2009). Radial magnetic field is estimated using cosine correction on LOS field. Partial magnetograms of AR8038 with radial field, $B_{r}$, are then cropped for analysis. The unsigned magnetic flux of an active region and that at the flaring PIL are related to ts activity, as previously found (Leka and Barnes, 2007; Schrijver, 2009; Welsch et al., 2009). We calculate the sum of unsigned flux of the entire AR frame as in Figure 6 (left) and that of the flaring PIL-region 


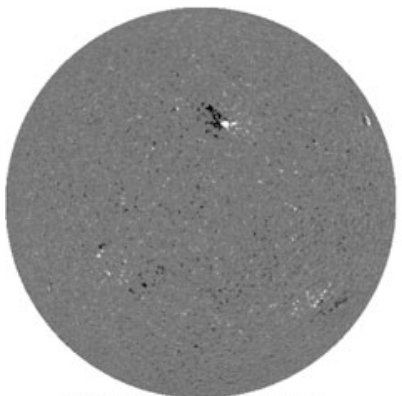

1997-05-12 01:40:04

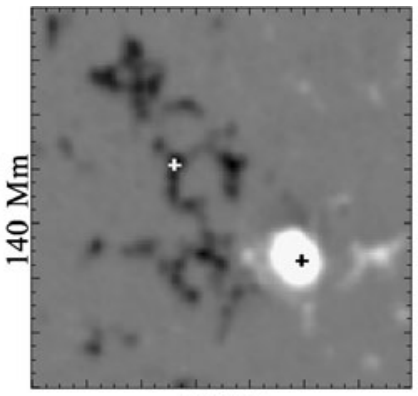

$140 \mathrm{Mm}$

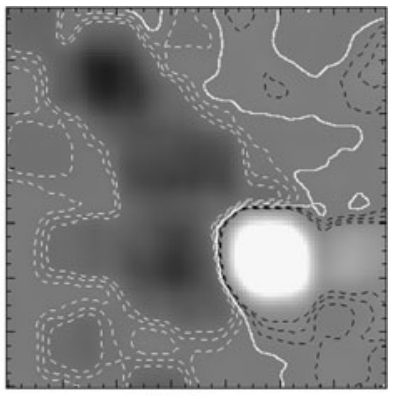

$140 \mathrm{Mm}$

Figure 5 Left: a sample MDI full disk magnetogram on 12 May 1997, white (black) for positive (negative) field. Center: a zoom-in magnetogram showing details of AR8038, and a movie of the full sequence (ar8038mag.gif) is attached. Black (white) cross symbol marks the positive (negative) weighted flux center. Right: smoothed magnetogram overlaid with selected contours to show the overall configuration of AR8038, where the long solid white line represents the PIL, and a movie of the full sequence (ar8038cont.gif) is attached.

shown as red contours over the magnetogram. The flaring PIL-region is obtained using a procedure described in Welsch et al. (2009) and Welsch and Li (2008) following a method proposed in Schrijver (2007). Here we use slightly modified parameters as specified in these steps: $i$ ) create positive and negative bitmaps of the AR frame corresponding to $\left|B_{r}\right|>70 \mathrm{G}$ (gauss); $i$ ) dilate the bitmaps by three pixels and compute the product of dilated positive and negative bitmaps to find overlapping pixels; iii) compute a PIL-weighting function, $W_{\text {PIL }}$ (red contours in Figure 6 left), by convolving the product bitmap with a normalized Gaussian of $7 \mathrm{Mm}$ FWHM. The defined PIL-region contains the flare site and does not include the full PIL of AR8038, but only the portion between stronger field. We then compute the total unsigned flux of the $W_{\text {PIL }}$ weighted AR magnetogram.

The sum of unsigned magnetic flux of the entire AR frame, $\Phi_{\mathrm{AR}}$, is plotted against time in black line in Figure 6 (right), and the sum of unsigned flux of the PIL-region, $\Phi_{\mathrm{PIL}}$, is the red line. $\Phi_{\mathrm{AR}}$ decreases monotonically by approximately $18 \%$ during $66 \mathrm{hrs}$ before the $\mathrm{C} 1.3$ flare. The radial flux decrease likely involves a few different processes commonly found after the emerging phase of an active region (Schrijver and Harvey, 1994; van DrielGesztelyi, 1998; Li and Welsch, 2008), including flux cancellation at the PIL. It has been widely appreciated that flux cancellation at the PIL of a magnetic region may also play an essential role in solar eruptions. Flux cancellation in AR8038 has been reported in different context as quoted earlier (Mathew and Ambastha, 2000; Liu, 2004; Li et al., 2004) and can be readily observed by readers at the PIL in our animation attachment (ar8038mag.gif). However, temporal flux variation rate of the active region as reported above, i.e., about $18 \%$ during 66 hours, are not previously measured elsewhere in continuous magnetogram sequence over several days prior to the eruption.

The role of radial flux decrease in eruptions may involve one or all of the following processes. If the radial field in the large part of the AR away from the flaring PIL is effectively the overlying field restraining the erupting structure, its decrease may imply the weakening of the 'tether'-like field. Some radial field may be transferred to transverse field through reconnection, which can increase magnetic shear or free energy. Welsch (2006) argued that magnetic flux cancellation increases the magnetic free energy in the system by reducing the magnetic potential energy. Linker et al. (2001, 2003) found in MHD simulations that with sufficient amount of flux cancellation/reduction (e.g., at cases, by about 

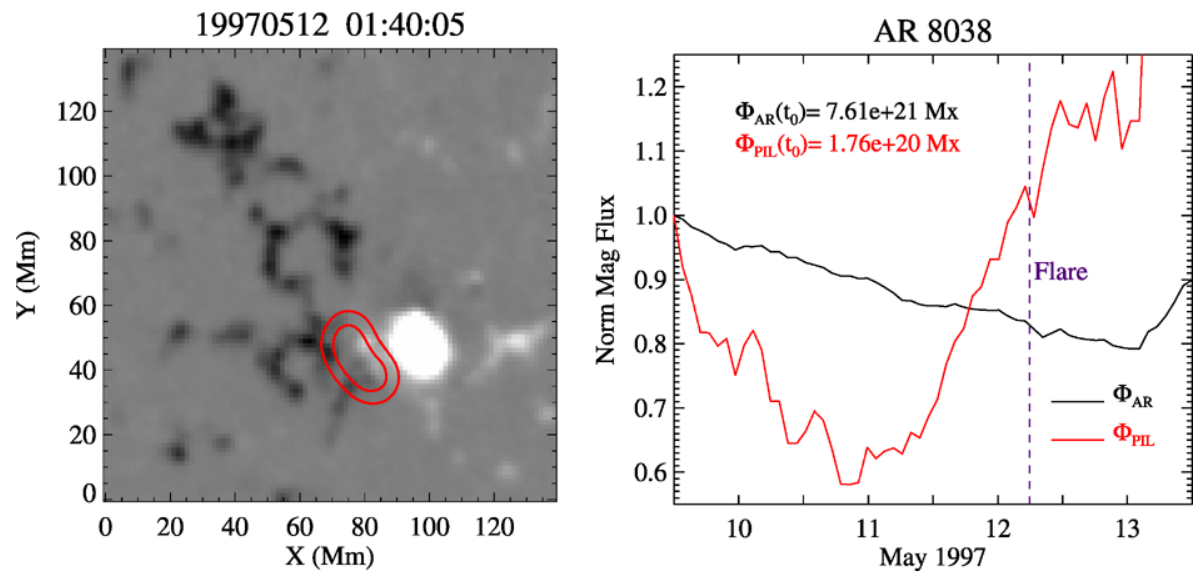

Figure 6 Left: MDI AR8038 magnetogram at 01:40:05, 12 May 1997. The red contour defines the PIL-region (see text for details). Right: $\Phi_{\mathrm{AR}}$ (black line) is the unsigned flux of the entire AR frame. $\Phi_{\mathrm{PIL}}$ (red line) is the unsigned flux of the PIL-region as defined by the red contours. The C1.3 flare time is marked as a purple dashed line.

$20 \%$ ), an eruption can be initiated in a single magnetic flux system containing stressed field at the PIL. This is not inconsistent with our observational results here.

The temporal evolution of $\Phi_{\mathrm{PIL}}$ is more complex as shown by the red line. It reflects a combination of flux cancellation and network field emergence (Mathew and Ambastha, 2000; Liu, 2004), as well as flux transported into the PIL-region by motions shown as converging flows in Li et al. (2004) and next section. After a decrease of about $40 \%$ during the first $30 \mathrm{hrs}, \Phi_{\mathrm{PIL}}$ increases by approximately $50 \%$ in the next 36 hours before the $\mathrm{C} 1.3$ flare. Schrijver (2007) reported a strong correlation between major flares ( $\mathrm{X}$ and $\mathrm{M}$ class) and the total unsigned flux $R$ of strong-field, high-gradient PIL-regions, where the field above a higher threshold of $150 \mathrm{G}$. They argued that greater $R$ values represent larger free magnetic energy. We relaxed the field threshold to accommodate this weaker magnetic region having maximum a C1.3 flare to calculate $\Phi_{\mathrm{PIL}}$ equivalent to the $R$ value. The increasing flux $\Phi_{\mathrm{PIL}}$ represents the increase of free magnetic energy and field gradient in the flaring PIL-region. While the persistent increase of $\Phi_{\mathrm{PIL}}$ leading to this moderate flare supports his argument, Schrijver (2007) did not study the temporal evolution prior to flares.

\subsection{Photospheric Flows in AR8038}

The LCT technique deduces magnetic-field-related motions by treating the magnetic field coherently rather than as individual features and obtains systematic horizontal flow field for each pixel (grid point) and has the potential to serve as input to numerical simulations for data-driven CME predictions. Here, using the foreshortening-corrected radial magnetic field and improved FLCT procedure (Welsch et al., 2007, 2009; Fisher and Welsch, 2008) we analyze the flow field of AR8038.

Figure 7 (left) shows one frame flow field between the magnetogram pair at 03:16:05 UT and 01:40:05 UT on 12 May before the flare. The FLCT flow speed in AR8038 ranges from 0 to $292.8 \mathrm{~m} \mathrm{~s}^{-1}$ with a mean value of $63.2 \mathrm{~m} \mathrm{~s}^{-1}$ and median $57.5 \mathrm{~m} \mathrm{~s}^{-1}$. The flow field has a persistent converging flow from the diffuse negative field towards the flaring PIL next to the sunspot that remains a similar speed during our four-day interval. There is also 

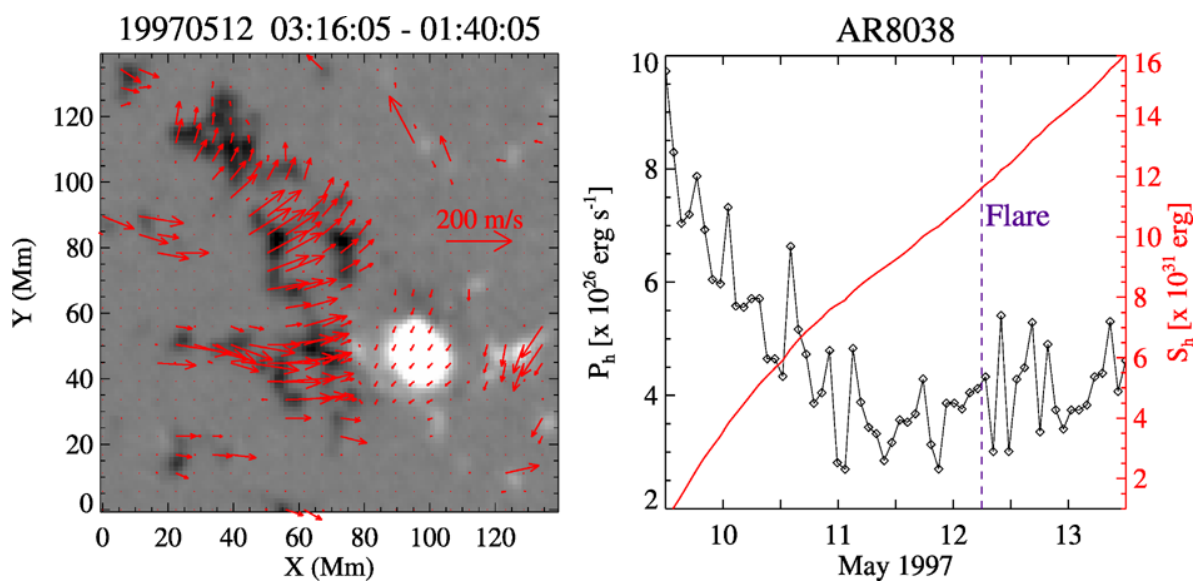

Figure 7 Left: a sample FLCT flow field on 12 May 1997. Right: the proxy Poynting flux $P_{\mathrm{h}}$ is shown in black-line-connected diamonds, the integrated proxy Poynting flux $S_{\mathrm{h}}$ is shown in a red line, and the C1.3 flare time is marked as a purple dashed line.

an northward flow in the diffuse negative field farther away from the flaring PIL with a speed that decreases over time. Being consistent with the observed flow field, the magnetic field distribution of AR8038 becomes more elongated or stretched over time in the northsouth direction. The AR tilt angle increased by about $11^{\circ}$ during the four days. The angle between the line connecting the positive and negative flux centers (shown as cross symbols in Figure 5 center) and the local latitude gives the tilt angle of the region. Although we did not find a significant concentrated shear flow along the PIL, there may exist an effective large-scale shear distributed in the AR that can stress the field to increase the free energy. While triggers of flares and CMEs may be small-scale features, the free energy that eruptions release may be stored in larger-scale magnetic regions.

Welsch et al. (2009) found a good correlation between the flare activity and a 'proxy Poynting flux' of an AR and argued that the proxy could give a crude order-of-magnitude estimate of energy flux input to the corona using LOS field observations. The proxy, referred to as $P_{\mathrm{h}}$ here, is defined in the following equation

$$
P_{\mathrm{h}}=\sum u B_{r}^{2} \mathrm{~d} A
$$

where $u$ is the horizontal flow field, $B_{r}$ is the radial magnetic field, and $d A$ is the area of each MDI pixel. Dimensionally, $P_{\mathrm{h}}$ has units of an energy flux, $\mathrm{erg} \mathrm{s}^{-1}$; see Welsch et al. (2009) for further details.

Based on the FLCT-estimated flow field, we calculate $P_{\mathrm{h}}$ for each frame of AR8038 magnetogram, which is plotted in Figure 7 (right) in black-line-connected diamonds. We further calculate the sum over time, $t$, as follows,

$$
S_{\mathrm{h}}(t)=\sum_{t} P_{\mathrm{h}}(t) \mathrm{d} t
$$

$S_{\mathrm{h}}(t)$ in erg measures the integrated proxy Poynting flux from time 0 to $t$ as a variable. We here integrated the absolute value of $P_{\mathrm{h}}$, which is justified by the fact that both $B_{r}$ and $u$ do not abruptly reverse in time domain. $S_{\mathrm{h}}(t)$ is plotted in Figure 7 (right) in a red line. The total energy input to the corona indicated by this proxy is approximately $1.15 \times 10^{32} \mathrm{erg}$ during 

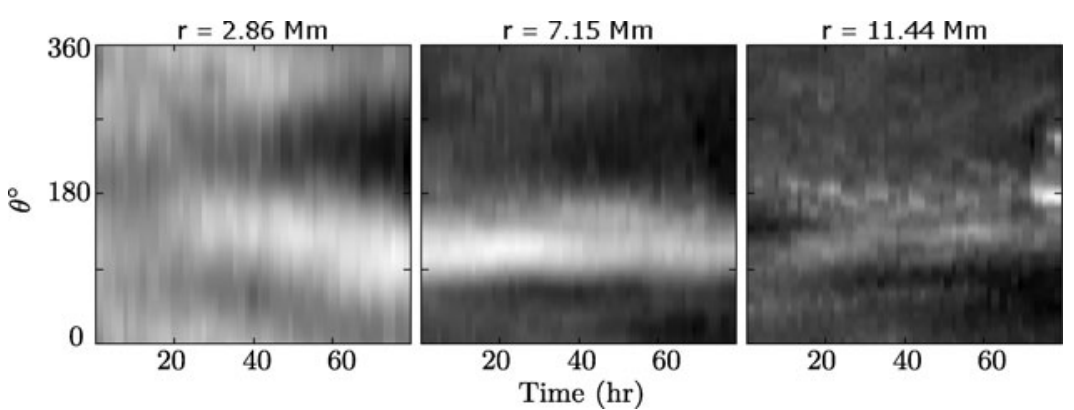

Figure 8 Stack plots of magnetic field in polar coordinate with origin at the sunspot center, made by stacking constant-radius strips from a time series of magnetograms (12:52, 9 May 1997 to 12:52, 13 May 1997). Three sample stack plots are shown at radial distances $r=2.86,7.15$ and $11.44 \mathrm{Mm}$ from left to right, respectively.

the $66 \mathrm{hrs}$, about half of the time from the potential state after the previous eruption to the C1.3 flare on 12 May. It suggests that sufficient energy for a flare/CME can be introduced through these flows deduced from photospheric magnetic field motions persisting for several days.

\subsection{Sunspot Rotation}

The FLCT results of the previous section did not show any significant shear flows in the vicinity of the PIL. However LCT methods might underestimate flows that are parallel to magnetic contours (Fisher and Welsch, 2008). In the strong positive field within the circular sunspot region, any rotational motion or circular flow patterns that preserve the sunspot flux distribution would be particularly difficult to detect. We here apply an alternative analysis to our magnetogram sequence that attempts to uncover any contour-preserving shear flow which is an efficient motion for driving a potential field into a non-potential state as is often conveniently used in simulation of CMEs (Linker et al. 2001, 2003; Gibson et al., 2004; Lynch et al., 2008).

We adapt an approach by Brown et al. (2003) to the magnetogram data to measure the rotation in the compact leading polarity sunspot. For each magnetogram in the sequence, we 'uncurl' the spot about its center (defined as the maximum of $\left|B_{r}\right|$ in the spot) by transforming $^{1}$ the data from $x-y$ Cartesian coordinates to $r-\theta$ polar coordinates. To determine field strengths on the $(r, \theta)$ gridpoints from the $(x, y)$ data, we use cubic-convolution interpolation (Park and Schowengerdt, 1983).

Once the magnetogram data are in polar coordinates, we construct angle-time plots at various radial distances from the spot center by extracting constant-radius strips (one pixel per strip at $1.43 \mathrm{Mm}$ ) from every image in the time sequence. The composite stack plots have $\theta$ along the vertical axis and time along the horizontal axis. In this form, rotational motions at a fixed radius appear as linear trends in the $\theta-t$ plane with positive (negative) slope indicating clockwise (counterclockwise) rotation.

Figure 8 shows three sample stack plots at radial distances $r=2.86,7.15$ and $11.44 \mathrm{Mm}$ from left to right, respectively. There is evidence of a minor clockwise rotation about $1^{\circ} \mathrm{h}^{-1}$ that persisted for approximately $30 \mathrm{~h}$ at the innermost radius (2.86 $\mathrm{Mm}$ from spot center).

\footnotetext{
${ }^{1}$ The routines developed for Cartesian-to-polar and polar-to-Cartesian conversion are available online at http://solarmuri.ssl.berkeley.edu/ fisher/public/software/idl/polar-cartesian/.
} 
No significant angular motion can be observed at any larger radii. Therefore, in this case, sunspot rotation does not appear to be a major source of free magnetic energy for flare/CME generation.

\section{Discussion and Conclusions}

We have shown that the three sequential CMEs from AR8038 can be considered homologous. Thus, the well-studied 12 May $1997 \mathrm{CME}$ is one of three recurrent events, which imposes a time constraint on its pre-eruption energy build-up process. To recall, all three CMEs had moderate speed, were associated with small flares and similar EIT double dimmings. The SXR sigmoid showed 'sigmoid to arcade to sigmoid' transformations interpreted as a repeatable free energy store - release - restore process. It was also shown that the corona relaxed to a nearly identical potential field state after the energy release by each eruption, and may serve to pre-condition the corona to a similar starting point for the free energy to re-build.

The process for the magnetic field to evolve from a potential state or near potential state to the next eruption has a time scale on the order of several days for this decaying small active region. What process restores the free energy (re-stresses the magnetic field) after each release? We analyzed the continuous MDI magnetogram sequence available for a substantial part of the time interval bounded by the adjacent homologous CMEs on 5 and 12 May, when the Sun and AR8038 were otherwise inactive between those CMEs. We argue that our measurements of magnetic flux evolution and a proxy Poynting flux in AR8038 may give some crude estimates, or serve as some lower bound measures for answering the above question. Our study differs from previous related ones by focusing on the magnetic free energy stored in the active-region-scale source region of the CME, rather than smaller scale features as triggers of the eruption.

Our analysis of magnetic field evolution confirms some previous findings of the processes in AR8038 leading to the C1.3 flare and halo CME, including flux cancellation and gradient increase at the flaring PIL (Mathew and Ambastha, 2000; Li et al., 2004; Liu, 2004). We have also taken further measures of temporal variations of the magnetic flux in the entire active region ( $\left.\Phi_{\mathrm{AR}}\right)$ and at the flaring PIL-region $\left(\Phi_{\mathrm{PIL}}\right)$ defined using a reproducible procedure. We showed, while the active region flux $\Phi_{\text {AR }}$ monotonically decreased by about $18 \%$ during $66 \mathrm{~h}$ prior to the $\mathrm{C} 1.3$ flare, the flux $\Phi_{\mathrm{PIL}}$ in the flaring PIL-region had more complex variations reflecting a combination of processes near the flaring PIL (details described in Section 3.1), but increased persistently by about $50 \%$ in the $36 \mathrm{hrs}$ prior to the C1.3 flare. The quantity $\Phi_{\text {PIL }}$ provides a measure of the free magnetic energy of a magnetic region (Schrijver, 2007; Welsch et al., 2009). The increase of $\Phi_{\mathrm{PIL}}$ shows evidence that the free energy is building up during the time leading to the eruption. However, temporal variation measurements similar to ours in continuous magnetogram sequence of several days are not reported previously for quantitative comparisons. We plan to apply our procedure to a large number of active regions to further evaluate the significance of these values in causing flares and CMEs, and whether and how these measures can be applied for predictions of solar eruptions.

The integrated proxy Poynting flux $S_{\mathrm{h}}$ defined in Equation (2) may give an order-ofmagnitude measure of the energy input to the corona. Our estimate yielded approximately $1.15 \times 10^{32} \mathrm{erg}$ during the $66 \mathrm{hrs}$ before the $\mathrm{C} 1.3$ flare, based on the FLCT flows and radial magnetic field. Given this estimate does indeed represent the order of the energy input to the corona, sufficient energy for a flare/CME can be introduced to the corona in several days 
through these magnetic field and flow quantities measured at the photosphere in this small active region.

Titov et al. (2008) found, in their magnetic field modeling, no breakout-null-point, but equivalent separators in the corona between the AR field and background field, where current layer may form to facilitate magnetic reconnection. They argued, however, that the driver of the eruption is more likely the flux cancellation at the photospheric PIL instead of the reconnection in the coronal current layer for the 12 May event. A global 3D MHD simulation of the 12 May 1997 event based on an MDI photospheric synoptic map has successfully initiated an eruption and the publication is currently in preparation (Linker et al., in preparation). The MHD model uses MDI photospheric synoptic map as boundary and initial conditions, and prescribed flows and flux cancellation to evolve and energize the corona to eventually initiate the eruption. The MDI synoptic map is constructed using MDI full disk magnetograms, which are the same data analyzed in this paper. Upon further details from their publication, the comparison between our observational results and the magnetic field evolution and energetics of the eruption in their simulation may provide further insights and/or constraints of the eruption process in this particular region.

We note that having concluded that the photospheric magnetic field motions could input sufficient energy to the corona, the magnetic evolution process cannot be fully explained using only LOS magnetograms. It remains a possibility that the magnetic free energy driving the eruptions is generated beneath the photosphere and partially released by each eruption (Gibson and Fan, 2006, 2008; Ranns et al., 2000; Nitta and Hudson, 2001; Zhang and Wang, 2002). This possible scenario could be supported if the converging flow toward the PIL in this decaying region is an indication of a still emerging U-loop (Magara, 2006, 2007) representing the field below the axis of a fluxrope at the core of the otherwise decaying and partially diffuse AR.

Evidently, even a simple magnetic region involves complex processes. We intend to further study magnetic field evolution leading to solar eruptions using improved data, e.g., vector magnetogram sequences from SDO in the near future.

Acknowledgements Y. Li and BTW are under the support of grant NSF ATM-0451438. Y. Li, BJL, JGL, and Y. Liu are under the support of NSF CISM ATM-0120950. Y. Li, JGL, and BJL are also partially supported by NASA SRT award NNG06GE51G. Major data sources are SOHO MDI EIT LASCO, MWO synoptic maps, and Yohkoh SXT. SOHO is a project of international cooperation of ESA and NASA. The authors thank Hugh Hudson for stimulating discussions.

Open Access This article is distributed under the terms of the Creative Commons Attribution Noncommercial License which permits any noncommercial use, distribution, and reproduction in any medium, provided the original author(s) and source are credited.

\section{References}

Antiochos, S.K., DeVore, C.R., Klimchuk, J.A.: 1999, Astrophys. J. 510, 485.

Arge, C.N., Luhmann, J.G., Odstrcil, D., Schrijver, C.J., Li, Y.: 2004, J. Atmos. Solar-Terr. Phys. $66,1295$.

Attrill, G.D.R., van Driel-Gesztelyi, L., Dmoulin, P., Zhukov, A.N., Steed, K., Harra, L.K., Mandrini, C.H., Linker, J.: 2008, Solar Phys. 252, 349.

Brown, D.S., Nightingale, R.W., Alexander, D., Schrijver, C.J., Metcalf, T.R., Shine, R.A., Title, A.M., Wolfson, C.J.: 2003, Solar Phys. 216, 79.

Canfield, R.C., Hudson, H.S., McKenzie, D.E.: 1999, Geophys. Res. Lett. 26, 627.

Canfield, R.C., Hudson, H.S., Pevtsov, A.A.: 2000, IEEE Trans. Plasma Sci. 28, 1786.

Crooker, N.U., Webb, D.F.: 2006, J. Geophys. Res. 111, A08108.

Fisher, G.H., Welsch, B.T.: 2008, In: Howe, R., Komm, R.W., Balasubramaniam, K.S., Petrie, G.J.D. (eds.) Subsurface and Atmospheric Influences on Solar Activity, ASP Conf. Ser. 383, 373. 
Fisher, G.H., Fan, Y., Longcope, D.W., Linton, M.G., Pevtsov, A.A.: 2000, Solar Phys. 192, 119.

Forbes, T.G., Linker, J.A., Chen, J., Cid, C., Kota, J., Lee, M.A., et al.: 2006, Space Sci. Rev. 123, 251.

Gibson, S.E., Fan, Y.: 2006, Astrophys. J. Lett. 637, 65.

Gibson, S.E., Fan, Y.: 2008, J. Geophys. Res. 113, A09103.

Gibson, S.E., Fan, Y., Mandrini, C., Fisher, G., Demoulin, P.: 2004, Astrophys. J. 617, 600.

Green, L.M., Kliem, B.: 2009, Astrophys. J. Lett. 630, 97.

Hudson, H.S., Lemen, J.R., St. Cyr, O.C., Sterling, A.C., Webb, D.F.: 1998, Geophys. Res. Lett. $25,2481$.

Leka, K.D., Barnes, G.: 2007, Astrophys. J. 656, 1173.

Leka, K.D., Canfield, R.C., McClymont, A.N., Van Driel Gesztelyi, L.: 1996, Astrophys. J. $462,547$.

Li, Y., Luhmann, J.G.: 2006, Astrophys. J. 648, 732.

Li, Y., Welsch, B.T.: 2008, In: Howe, R., Komm, R.W., Balasubramaniam, K.S., Petrie, G.J.D. (eds.) Subsurface and Atmospheric Influences on Solar Activity, ASP Conf. Ser. 383, 397.

Li, Y., Luhmann, J.G., Fisher, G.H., Welsch, B.T.: 2004, J. Atmos. Solar-Terr. Phys. 66, 1271.

Linker, J.A., Lionello, R., Mikić, Z., Amari, T.: 2001, J. Geophys. Res. 106(A11), 25165.

Linker, J.A., Mikić, Z., Lionello, R., Riley, P., Amari, T., Odstrcil, D.: 2003, Phys. Plasmas $10,1971$.

Linker, J.A., Mikić, Z., Lionello, R., Riley, P., Titov, V.: 2006, AGU Fall Meeting, SH33B-414.

Linker, J.A., Lionello, R., Mikić, Z., Titov, V., Riley, P.: 2008, AGU Spring Meeting, SP31D-05.

Liu, Y.: 2004, J. Atmos. Solar-Terr. Phys. 66, 1283.

Liu, C., Lee, J., Yurchyshyn, V., Deng, N., Cho, K., Karlický, M., Wang, H.: 2007, Astrophys. J. 669, 1372.

Lynch, B.J., Antiochos, S.K., DeVore, C.R., Luhmann, J.G., Zurbuchen, T.H.: 2008, Astrophys. J. 683, 1192.

Mathew, S.K., Ambastha, A.: 2000, Solar Phys. 197, 75.

Moore, R.L., Sterling, A.C., Hudson, H., Lemen, J.R.: 2001, Astrophys. J. 552, 833.

Magara, T.: 2006, Astrophys. J. 653, 1499.

Magara, T.: 2007, In: Shibata, K., Nagata, S., Sakurai, T. (eds.) New Solar Physics with Solar-B Mission, ASP Conf. Ser. 369, 367.

Nitta, N.V., Hudson, H.S.: 2001, Geophys. Res. Lett. 28, 3801.

Park, S., Schowengerdt, R.: 1983, Graph. Image Proc. 23, 256.

Plunkett, S.P., Thompson, B.J., Howard, R.A., Michels, D.J., St. Cyr, O.C., Tappin, S.J., Schwenn, R., Lamy, P.L.: 1998, Geophys. Res. Lett. 25(14), 2477.

Qiu, J., Hu, Q., Howard, T.A., Yurchyshyn, V.B.: 2007, Astrophys. J. 659, 758.

Ranns, N.D.R., Harra, L.K., Matthews, S.A., Culhane, J.L.: 2000, Astron. Astrophys. 360, 1163.

Scherrer, P.H., Bogart, R.S., Bush, R.I., Hoeksema, J.T., Kosovichev, A.G., Schou, J., et al.: 1995, Solar Phys. 162, 129.

Stenborg, G., Vourlidas, A., Howard, R.A.: 2008, Astrophys. J. 674, 1210.

Schrijver, C.: 2007, Astrophys. J. Lett. 655, 117.

Schrijver, C.: 2009, Adv. Space Res. 43, 739.

Schrijver, C.J., Harvey, K.L.: 1994, Solar Phys. 150, 1.

Sterling, A.C., Hudson, H.S., Thompson, B.J., Zarro, D.M.: 2000, Astrophys. J. 532, 628.

Thompson, B.J., Plunkett, S.P., Gurman, J.B., Newmark, J.S., St. Cyr, O.C., Michels, D.J.: 1998, Geophys. Res. Lett. 25, 2465.

Titov, V.S., Mikic, Z., Linker, J.A., Lionello, R.: 2008, Astrophys. J. 675, 1614.

van Driel-Gesztelyi, L.: 1998, In: Alissandrakis, C.E., Schmieder, B. (eds.) Three-Dimensional Structure of Solar Active Regions, ASP Conf. Ser. 155, 202.

Webb, D.F., Cliver, E.W., Crooker, N.U., St. Cyr, O.C., Thompson, B.J.: 2000, J. Geophys. Res. $105,7491$.

Welsch, B.T.: 2006, Astrophys. J. 638, 1101.

Welsch, B.T., Li, Y.: 2008, In: Howe, R., Komm, R.W., Balasubramaniam, K.S., Petrie, G.J.D. (eds.) Subsurface and Atmospheric Influences on Solar Activity, ASP Conf. Ser. 383, 429.

Welsch, B.T., Longcope, D.W.: 2003, Astrophys. J. 588, 620.

Welsch, B.T., Fisher, G.H., Abbett, W.P., Regnier, S.: 2004, Astrophys. J. 610, 1148.

Welsch, B.T., Abbett, W.P., DeRosa, M.L., Fisher, G.H., Georgoulis, M.K., Kusano, K., Longcope, D.W., Ravindra, B., Schuck, P.W.: 2007, Astrophys. J. 670, 1434.

Welsch, B.T., Li, Y., Schuck, P.W., Fisher, G.H.: 2009, Astrophys. J. 705, 821.

Yurchyshyn, V., Liu, C., Abramenko, V., Krall, J.: 2006, Solar Phys. 239, 317.

Zhang, J., Wang, J.: 2002, Astrophys. J. Lett. 566, 117.

Zhao, X.P., Plunkett, S.P., Liu, W.: 2002, J. Geophys. Res. 107(A8), SSH13-1. 